\title{
Metabolic Syndrome and Infertility in Women
}

\author{
Ahmed Al Awlaqi ${ }^{1}$, Khalid Alkhayat ${ }^{1}$, Mohamed E. Hammadeh ${ }^{1 *}$
}

\begin{abstract}
Polycystic ovary syndrome (PCOS) is a common endocrine disorder in women and it affects approximately $5 \%-8 \%$ of premenopausal women. Metabolic syndrome has been reported in the reproductive literature to fall under a cluster of endocrine disturbances, including hypertension, obesity, dyslipidemia, and insulin resistance. Literature findings have demonstrated that conditions of negative energy balance and metabolic stress, such as diabetes mellitus type 1, acute inflammation, and chronic dietary restriction can affect fertility. These conditions cause hypogonadism by suppressing the expression of the hypothalamic KiSS or kisspeptin. Diabetes affects reproductive function in women. The objective of the current review is to explore the correlation between metabolic syndrome and infertility in women. To achieve this, a review of literature studies between 2007 and 2015 was undertaken to evaluate current evidence-based practice on the topic. Keywords, such as metabolic disorders, women fertility, and reproduction were used to search for data from PubMed, MEDLINE, CINAHL, ERIC, and EMBASE databases. The inclusion and exclusion criteria was based on the appropriateness of the research design in reference of research objectives, risks of bias, statistical issues, quality reporting, choice of measures of outcome, quality of intervention, and studies conducted between 2007 and 2015. The results from the highest evidence available confirm that metabolic disorders have adverse impacts on the reproductive health of women, and specifically their fertility. Metabolic disorders like hyperlipidemia, obesity, and diabetes can directly or indirectly affect the fertility of women through the interruption of either the ovarian functions or the pituitary-hypothalamic functions. Furthermore, metabolic disorders increase the risks of cervical and endometrial cancers in women that hamper the reproductive health and fertility of women.

Keywords: Obesity, Metabolic, Endocrinology
\end{abstract}

\section{Introduction}

Three major interrelated abnormalities characterize metabolic syndrome: elevated blood pressure, dyslipidemia, and elevated blood plasma (1). The three abnormalities directly contribute to pro-inflammatory and pro-thrombic state, an outcome that predisposes an individual to the development of diabetes type 2 and atherosclerotic cardiovascular disease (2). Insulin resistance and hyperinsulinemia are the primary underlying metabolic abnormalities reported in metabolic syndrome and polycystic ovarian syndrome (3). Elevated circulating insulin levels and insulin resistance induces unfavorable changes in increased androgen production and lipid metabolism from theca cells (4). Having excess release of androgens can result in unfavorable metabolic problems resulting in central distribution of fat and dyslipidemia. Dyslipidemia and excess insulin in obese women may contribute to metabolic syndrome and polycystic ovarian syndrome (1). The android fat distribution pattern may be explained by hyperandrogenism, resulting in a vicious circle of central adiposity, hyperinsulinism, and metabolic abnormalities (1).

\section{Metabolic Disorder \\ Metabolic disorders are caused by a malfunction in body metabolism. Examples of metabolic disorders include obe- sity, diabetes, and dyslipidemia, which together constitute}

'metabolic syndrome' (see Figure 1). Metabolic syndrome, also called insulin resistance syndrome or syndrome $\mathrm{X}$ is a collection of metabolic abnormalities (5). It is condition typified by glucose intolerance, central obesity, dyslipidemia, and hypertension (6,7). Even though the term "metabolic syndrome" has been used to denote a collection of risk factors, there are debates concerning its existence, and various definitions have been used (8). The incidence of metabolic syndrome is on the rise globally (5). The terms metabolic disorder and metabolic syndrome will be used interchangeably in this discussion to refer to the same condition.

\begin{abstract}
Prevalence
The global prevalence of metabolic syndrome ranges from less than $10 \%$ to as high as $46 \%$. The prevalence is influenced by region, ethnicity, age, the interpretation of metabolic syndrome used and the environment $(9,10)$, as well as the diagnostic criteria used (11). Metabolic syndrome is a major public health problem, particularly in the developed nations such as the United States and Europe. The prevalence in the United States is reported to be $23 \%$ among nondiabetic individuals (8). The prevalence of metabolic syndrome among the European American ethnic groups in Europe ranges from $20 \%$ to $30 \%$ (7). Cohort studies conducted in Iran have shown that the prevalence
\end{abstract}

Received 13 January 2016, Accepted 14 May 2016, Available online 7 June 2016 
of metabolic syndrome is more than $30 \%$ and that it is significantly higher in women than in men $(23.1 \%$ women vs. $18.4 \%$ male) $(6,12)$.

\section{Pathogenesis}

The pathogenesis of metabolic syndrome is not completely clear; nonetheless, there are some factors that have been implicated, including dietary habits and sedentary lifestyles, increasing age and body mass index (7). For instance, factors like the history of maternal gestational diabetes, decreased parity, and postmenopausal status have been shown to increase the risk of metabolic syndrome in women (13). Recent studies have demonstrated that there are sex differences concerning risk predictors of metabolic syndrome, implying that levels of sex hormones and estrogen/androgen balance potentially play a vital role in determining metabolic syndrome $(14,15)$.

A section of earlier studies has postulated that men are likely to have a higher prevalence than age-matched premenopausal women are (13). Nonetheless, the relationship seems not to hold after menopause (16), with prevalence being significantly higher among women compared to men, particularly after the age of 60 (16). Kim et al (17) demonstrated postmenopausal status was linked to a higher metabolic syndrome risk independent of normal aging in Korean women. Cho et al (18) reported similar findings in Chinese subjects.

\section{Methodology}

The literature review for the metabolic disorder and fertility in women covered a period of eight years; that is from 2007 to 2015. The study articles were extracted from five medical databases, including CINAHL, MEDLINE, EMBASE, PubMed, and ERIC. The articles were researched using keywords like diabetes, reproductive health, metabolic disorders, body mass index, female fertility and metabolic diseases. Initial approach reviewed the journal abstracts for their research objectives and relevance. The inclusion criteria took into account the appropriateness of the research methodologies of the articles, the research objectives, statistical issues, risks of bias, the measures of outcomes, quality reporting, studies undertaken between 2007 and 2015, and the quality of intervention.

\section{Results}

Metabolic Disorder and Women's Fertility

Energy stores and metabolism in the body determine the onset of puberty and fertility, albeit the mechanism by which these occur, remains unclear (19-21). The current body of literature shows that conditions of negative energy balance and metabolic stress, such as diabetes mellitus type 1 in primates and rodents $(20,22)$ acute inflammation in rats, and chronic dietary restriction can affect fertility $(23,24)$. These conditions cause hypogonadism by suppressing the expression of the hypothalamic KiSS/ kisspeptin, implying that Kiss1 neurons are sensitive to alterations in metabolic status $(25,26)$.
According to Roa et al (26), acute administration of kisspeptin is sufficient to normalize the secretion of gonadotrophin in diabetic rats, and testosterone levels in diabetic males). Chronic treatment with kisspeptin-10 has also been shown to ameliorate significantly many longterm reproductive deficits in diabetics (27). The findings demonstrate that defective Kiss1 tone in the hypothalamus significantly contributes to the hypogonadotropic hypogonadism $(28,29)$, mostly seen in individuals with poorly controlled diabetes mellitus type 1 (30).

Besides, women who are obese and diabetic have been shown to have an elevated risk of complications associated with pregnancy (31). In particular, glycemic control has been associated with undesirable effects on the reproductive of women with both type 1 and type 2 diabetes mellitus. Uncontrolled diabetes has been associated with sexual dysfunction in women. It has also been associated with increased risk of morbidity and mortality in the offspring (32).

Pathophysiology of the Reproductive Axis in Patients With Metabolic Disorder

\section{Pituitary-Hypothalamic Function}

Earlier studies carried out in females have demonstrated that individuals with insufficient metabolic control and primary and secondary amenorrhea exhibit low levels of estradiol (30). The women also present insufficient Luteinizing hormone (LH) and follicle stimulating hormone (FSH), which have mostly been associated with a lack of residual insulin secretion (33). The poor metabolic control observed in a majority of these patients explain the perturbations (34). The hypogonadotropic hypogonadism seen in amenorrheic diabetic females has been shown to be similar to that linked to other metabolic stress forms, including strenuous exercise and anorexia nervosa (35). However, other reports have shown that diabetic patients with secondary amenorrhea due to hypogonadotropic hypogonadism failed to recover following theimprovement in metabolic control (36), implying that a certain group of diabetic patients is prone to hypogonadism (34).The hypothalamic origin of the decreased levels of gonadotropin in amenorrheic and diabetic patients has been explained $(25,26)$. Abnormalities of gonadotropin-releasing hormone $(\mathrm{GnRH})$ pulse generator are hypothesized on LH pulses studies, indicating the secretory activity of the GnRH neurons (37). Such studies have demonstrated a decrease in the number of LH pulses, wider pulses, and a decrease in pulse amplitude in patients with diabetes and amenorrhea relative to those with normal menstrual $\mathrm{cy}$ cles $(34,37)$.

A toxic effect of hyperglycemia on the neurons of the hypothalamus has been proposed by observations of reduced LH response to GnRH stimuli (34). The toxic effects have also been reported with increasing duration of diabetes and by GnRH secretion abnormalities and increased apoptosis in an immortalized GnRH cell line exposed to hyperglycemia (37). These findings are suggestive that chronic hyperglycemia is likely to trigger glucotoxicity 
in GnRH neurons; nonetheless, mediators of the central nervous system such as increased opioidergic activity, catecholamine levels, and dopaminergic tone have also been implicated in hypogonadism pathophysiology in diabetic patients (38).

\section{Ovarian Function}

The invention of modern intensive treatment for diabetes has been associated with reduced hypogonadism prevalence as demonstrated by the reduction in amenorrhea prevalence (30). Sadly, an increase in the incidence of reproductive abnormalities caused by insulin excess, particularly during polycystic ovary syndrome, excessive weight gain, and hyperandrogenism has been reported (34). Under normal physiological circumstances, the pancreas secretes insulin into the portal circulation, and the liver eliminates a significant fraction of the secreted insulin (39).

In patients with diabetes type 1 , insulin is usually administered into the subcutaneous tissue from where it is absorbed into the systemic circulation, omitting the hepatic first-pass step and consequently exposing the peripheral tissues to supra-physiologic levels of insulin (33).The significance of insulin action on human reproductive function is underscored by insulin receptor expression in several tissues, including ovaries, uterus, hypothalamus, and pituitary (40). Insulin promotes ovarian steroidogenesis and follicular development through insulin receptors in granulosa cells. Insulin has also been shown to augment FSH-stimulated steroid secretion (41). Additionally, its gonadotropic effects on folliculogenesis promote recruitment and pre-ovary follicular growth. These numerous actions of insulin form the basis for the potential impact of disturbed insulin secretion on the development and functions of the ovaries $(34,41)$.

Hyperglycemia also affects reproductive function in women. Elevated blood glucose triggers peripheral insulin resistance. Existing insulin resistance and hyperinsulinemia have been shown to cause polycystic ovary syndrome (PCOS) (42). Hyperglycemia is also thought to affect ovarian function via the presence of advanced glycation products and receptors. These receptors have been reported to exist in theca and granulosa cells of healthy women (43). Few studies have investigated ovulatory function in diabetic women. Some have reported delays in ovulation in women trying to become pregnant, implying that such women have a longer follicular phase (44-46).

Codner and colleagues conducted a prospective study on ovulation in non-hyperandrogenic adolescents with type 1 diabetes $(\mathrm{n}=31)$ and compared them with a control group $(n=52)$. They found out that the diabetic girls did not experience a decrease in ovulation. They found similarity in the fraction of ovulatory cycles in both the treatment and control groups (34.5\% and 36.3\% respectively). Metabolic control was found to have a slight effect on the rate of ovulation. An increased rate of ovulation every 100 days and a higher percentage of ovulatory cycles were reported in diabetic girls to optimal metabolic control that diabetic girls with insufficient control (25).

Even though these data are suggestive of preserved ovulation, most studies have reported fewer pregnancies and live births in women with diabetes mellitus type 1 (47). Fecundability has been reported to be lower in diabetic women, and this has been associated with sexual dysfunction in such women. Reports show that diabetic women experience sexual dysfunction, particularly dyspareunia, decreased desire, as well as changes in the arousal phase, which have been associated with depressive symptoms and marital problems (48). Studies evaluating fertility treatments on diabetic women agree that the main factor in ensuring successful pregnancy is achieving optimal metabolic control before using more invasive techniques (30). In vitro fertilization treatments in women with type 1 diabetes have been shown to have similar results to those in non-diabetic women when optimal metabolic control is achieved $(2,34,49)$.

\section{Obesity and Women's Fertility}

Lifestyle factors can have negative or positive effects on women's fertility. Obesity, which is a component of metabolic syndrome, has been shown to have a significant impact on female fertility. Reports show that there is a high prevalence of obese women in the infertile population. Similarly, obesity has been linked to infertility (50). Obese women mostly have decreased insulin sensitivity putting them at an increased risk of several adverse outcomes in pregnancy. Metabolic syndrome is associated with increased risk of inflammation, metabolic disturbances of nutrient metabolism, and hypertensive disorders during pregnancy.

Even though these conditions can be resolved clinically, the woman remains at risk of metabolic syndrome in later life $(51,52)$. Obese women have been shown to have a $10 \%$ $15 \%$ increased risk of preeclampsia, which has been associated with poor pregnancy outcomes (53). Obese women have also been shown to have lower sex hormone-binding globulin concentrations than lean women (51). Furthermore, obese women have reduced fertility about women with normal body weight in both conceptions achieved by assisted reproduction techniques and natural conception. The subfertility in obese women is partly ascribed to the complete absence or reduced frequency of ovulation (53). Obesity has been reported to have a negative impact on assisted reproduction techniques because it adversely affects the duration of stimulation of the ovaries, fertilization rate, quality, and some oocytes and embryos, the rate of embryo transfer, and pregnancy rates (54). Consequently, obese women have been shown to require higher doses of gonadotropins and longer durations of ovarian stimulation. Higher incidence of follicular synchrony and higher cancellation rates has also been reported (55).

The lower number of oocytes retrieved in obese women about normal weight women could be attributed technical difficulties or lower number of follicles (56). Furthermore, obesity can hinder accurate transabdominal ultrasonographic imaging during embryo transfer. These mecha- 
nisms explain the decrease in implantation and rates of pregnancy in obese women undergoing assisted reproduction techniques. Overweight and obesity have also been associated with menstrual irregularities and reduced rate of conception. Also, it increases the risk of miscarriage and contributes to perinatal and maternal complications (57).

\section{Diabetes and Women's Fertility}

Hyperglycemia and insulin resistance have been shown to induce oxidative stress resulting in an imbalance between free oxygen radical activity and antioxidant defense mechanisms at the cellular level (58). Oxidative stress is also postulated to deteriorate fetal development (59). Studies conducted on a female and male animals have demonstrated that uncontrolled diabetes is likely to result in a profound hypogonadotropic state, typified by low basal levels of sex steroids and gonadotropins, defective gonadotropin responses to gonadectomy, and reduced LH pulsatility, resulting in disturbed negative-feedback responses (60).

Positive-feedback disruption in females has been shown to cause delayed or absent preovulatory LH surges and anovulation in females. Reports have suggested that some of the reproductive deficits associated with diabetes in women could result from ovarian alterations $(61,62)$. Follicular growth and survival abnormalities, including increased granulosa and follicular apoptosis, as well as impaired oocyte-granulosa communication, oocyte maturation, and development of ovarian follicles have been observed in diabetic animal models. Ovarian steroidogenesis perturbation and ovulation have also been observed in female diabetic mice (63).

Furthermore, insulin deficiency has been linked to defective ovulation, and this can be reversed through treatment with insulin in diabetic rodents. Ovarian protein glycation has been described in non-diabetic mice and hamsters (65) and has the likelihood of affecting ovarian aging in mice (65). These observations are suggestive of the direct harmful effects of hyperglycemia and low insulin levels on ovarian functions, which in turn have a direct impact on women's fertility $(66,67)$.

Metabolic Disorders and Reproductive Cancers in Women Reports show that there is a direct correlation between metabolic disarrangements seen in metabolic syndrome and carcinogenesis (68). Cancers affecting the reproductive organs such as the uterus have anegative impact on a woman's fertility because the uterus plays a key role in accommodation the fetus. Carcinogenesis is associated with lifestyle factors, with excess weight gain being acknowledged as a major risk factor accounting for almost half of endometrial cancer cases in industrialized countries (69). Metabolic disorders such as obesity and diabetes are thought to increase the risk of developing reproductive health cancers such as endometrial or breast cancer (70). Kurdoglu et al (63) reported that oxidative stress characterized by decreased antixidant and increased oxidant levels that are independent of insulin resistance may play a role in the pathogenesis of polycystic ovarian syndrome in young non-obese women. Hyperinsulinemia, which is caused by hyperglycemia has been shown to facilitate carcinogenesis by increasing circulating free insulin-like growth factors-1 (IGF-1). People with elevated IGF-1 concentrations have been shown to have a higher risk of developing cancer (71). The pathways for insulin receptor activating signaling can trigger proliferation of cancer cells, protecting from apoptotic stimuli, metastasis, and invasion. It can also stimulate the proliferation of normal cells such as the smooth muscle cell and migration, which are required for cancer cell growth (72). Hyperglycemia and impaired regulation of glucose have also been shown to promote the growth of cancer cells (73). Cancer cell glucose transporting proteins have been shown to increase in an attempt to respond to the increased glucose demands (74).

Epidemiological studies have also shown that there is an association between diabetes and endometrial cancer (75). Similarly, hypertension has also been positively associated with an increased risk of endometrial cancer (76). On the other hand, data on the association between endometrial cancer and dyslipidemia is scarce (77). Friedenreich and colleagues (70) carried out a case-control study to examine the role of metabolic dysfunction in endometrial carcinogenesis in women with endometrial cancer $(n=515)$ in Alberta, Canada. Their study revealed that there was a clear association between metabolic syndrome and increased risk of endometrial cancer.

The association between metabolic syndrome and risk of cervical cancer has also been investigated. Hypertriglyceridemia has been shown to increase the risk of cervical cancer and thus can have an impact on women's fertility. Elevated triglycerides have been shown to promote the proliferation of cancer cells and their anti-apoptotic activity by causing oxidative stress and generating reactive oxygen species, which induce DNA damage $(78,79)$. Penranda and colleagues (65) conducted a case-control study to investigate the association between metabolic syndrome in women $(n=585924)$ aged 21 years and above in the United States. Their study demonstrated that there was increased odds of a history of cervical cancer among those subjects with metabolic syndrome (80). Also, cancers and their associated treatments, including chemotherapy, radiotherapy, surgery, biologic, and endocrine therapy have been shown to affect profoundly gonadal function and subsequently, women's fertility (81).

\section{Conclusion}

Reproduction is an important process in human lives. Metabolic disorders can have adverse effects on women's reproduction by affecting their fertility. Metabolic disorders, including diabetes, obesity, and hyperlipidemia can affect women's fertility directly or indirectly by interfering with the pituitary-hypothalamic function or ovarian function. Also, they increase the risk of reproductive cancers such as endometrial and cervical cancer in women. These 
factors affect women's reproductive health and interfere with women's fertility in particular.

Metabolic disorders cause hypogonadism through the suppression of the suppression of the hypothalamic KiSS/ kisspeptin expression. In diabetic patients, elevated blood glucose triggers peripheral insulin resistance. The existing insulin resistance and hyperinsulinemia can potentially trigger the development of PCOS. Hyperglycemia is also thought to affect ovarian function via advanced glycation products and receptors. Their receptors have been reported to exist in theca and granulosa cells of healthy women. The findings also indicate that obesity, which is a component of metabolic syndrome, has a significant impact on female fertility as evident from reports that show a high prevalence of obese women in the infertile population. Obese women mostly have decreased insulin sensitivity, putting them at an increased risk of several adverse outcomes in pregnancy.

Furthermore, the review has shown that metabolic disorders are thought to increase the risk of developing reproductive health cancers such as endometrial or breast cancer. Hyperinsulinemia, which is caused by hyperglycemia, has been shown to facilitate carcinogenesis by increasing circulating free insulin-like growth factors-1 (IGF-1). Hypertriglyceridemia has been shown to increase the risk of cervical cancer and thus can have an impact on women's fertility. Elevated triglycerides have been shown to promote the proliferation of cancer cells and their anti-apoptotic activity by causing oxidative stress and generating reactive oxygen species, which induce DNA damage.

\section{Ethical Issues}

Not applicable.

\section{Conflict of Interests}

The author has no conflicts of interest to disclose.

\section{References}

1. Amudha M, Rani S, Kannan K, Manavalan R. An updated overview on causes, diagnosis and management of infertility. International Journal of Pharmacy, Science and Revised Research. 2013;18(1):155-164.

2. Roa J, Aguilar E, Dieguez C, Pinilla L, Tena-Sempere M. New frontiers in kisspeptin/GPR54 physiology as fundamental gatekeepers of reproductive function. Front Neuroendocrinol. 2008;29:48-69.

3. Chaudhary S, Chaudhary A. Human Anatomy \& Physiology. Jalandhar S. Vikas \& Co; 2010:259.

4. Akter S, Jesmin S, Rahman MM, et al. Higher gravidity and parity are associated with increased prevalence of metabolic syndrome among rural bangladeshi women. PLoS One. 2013;8(8):e68319. DOI:10.1371/journal.pone.0068319.

5. Shalini M, Suresh BK, Srinivasa MA, Girish B, Mounika K, Vaishnavi B. Metabolic syndrome among urban and rural women population - a cross sectional study. J Clin Diagn Res. 2013;7(9):1938-1940. doi:10.7860/ JCDR/2013/6467.3363.

6. Hadaegh F, Hasheminia M, Lotfaliany M, Mohebi R, Azizi F, Tohidi M. Incidence of metabolic syndrome over 9 years follow-up; the importance of sex differences in the role of insulin resistance and other risk factors. PLoS One. 2013;8(9):e76304. doi: 10.1371/journal.pone.0076304.

7. Shahini N, Shahini I, Marjani A. Prevalence of metabolic syndrome in Turkmen ethnic groups in Gorgan. J Clin Diagn Res. 2013;7(9):1849-1851. doi:10.7860/ JCDR/2013/6035.3331.

8. Bjørge T, Stocks T, Lukanova A, et al. Metabolic syndrome and endometrial carcinoma. Am J Epidemiol. 2010;171(8):892-902. doi: 10.1093/aje/kwq006.

9. Arthur FK, Adu-Frimpong M, Osei-Yeboa J, Mensah FO Owusu L. The prevalence of metabolic syndrome and its predominant components among pre-and postmenopausal Ghanaian women. BMC Res Notes. 2013;6:446. DOI: 10.1186/1756-0500-6-446.

10. Kolovou GD, Anagnostopoulou KK, Salpea KD, Mikhailidis DP. The prevalence of metabolic syndrome in various populations. Am J Med Sci. 2007;333:362-371.

11. Salaroli LB, Saliba RA, Zandonade E, Molina Mdel C, Bissoli NS. Prevalence of metabolic syndrome and related factors in bank employees according to different defining criteria, Vitória/ES, Brazil. Clinics (Sao Paulo). 2013;68(1):69-74.

12. Zabetian A, Hadaegh F, Azizi F. Prevalence of metabolic syndrome in Iranian adult population, concordance between the IDF with the ATPIII and the WHO definitions. Diabetes Res Clin Pract. 2007;77(2):251-257.

13. Ortiz AP, Suárez E, Beauchamp G, Romaguera J, SotoSalgado M, Pérez CM. Correlates of the metabolic syndrome among a sample of women in the San Juan Metropolitan area of Puerto Rico. Metab Syndr Relat Disord. 2010;8(3):235242. doi:10.1089/met.2009.0076.

14. Agirbasli M, Agaoglu NB, Orak N, et al. Sex hormones and metabolic syndrome in children and adolescents. Metabolism. 2009;58:1256-1262. DOI:10.1016/j. metabol.2009.03.024.

15. Kim HA, Lee SY, Kwon HS, et al. Gender differences in the association of insulin resistance with metabolic risk factors among Korean adolescents: Korea National Health and Nutrition Examination Survey 2008-2010. Diabetes Res Clin Pract. 2013;99: 54-62. doi:10.1016/j.diabres.2012.10.011.

16. Liang $\mathrm{H}$, Chen $\mathrm{X}$, Chen $\mathrm{Q}$, et al. The metabolic syndrome among postmenopausal women in rural Canton: prevalence, associated factors, and the optimal obesity and atherogenic indices. PLoS One. 2013;8(9):e74121. doi:10.1371/journal. pone.0074121.

17. Kim HM, Park J, Ryu SY, Kim J. The effect of menopause on the metabolic syndrome among Korean women: The Korean National Health and Nutrition Examination Survey, 2001. Diabetes Care. 2007;30:701-706.

18. Cho GJ, Lee JH, Park HT, et al. Postmenopausal status according to years since menopause as an independent risk factor for the metabolic syndrome. Menopause. 2010;15: 524-529.

19. Hill JW, Elmquist JK, Elias CF. Hypothalamic pathways linking energy balance and reproduction. Am J Physiol Endocrinol Metab. 2008;294(5):E827-32. doi:10.1152/ ajpendo.00670.2007.

20. Castellano JM, Bentsen AH, Mikkelsen JD, Tena-Sempere, M. Kisspeptins: bridging energy homeostasis and reproduction. Brain Res. 2010;1364:129-138.

21. Roa J, Navarro VM, Tena-Sempere M. Kisspeptins in reproductive biology: consensus knowledge and recent developments. Biol Reprod. 2011;85(4):650-660. doi: 10.1095/biolreprod.111.091538. 
22. Kalamatianos T, Grimshaw SE, Poorun R, Hahn JD, Coen CW. Fasting reduces KiSS-1 expression in the anteroventral periventricular nucleus (AVPV): effects of fasting on the expression of KiSS-1 and neuropeptide $\mathrm{Y}$ in the AVPV or arcuate nucleus of female rats. J Neuroendocrinol. 2008;20:1089-1097.

23. Wahab F, Ullah F, Chan YM, Seminara SB, Shahab M. Decrease in hypothalamic Kiss1 and Kiss1r expression: a potential mechanism for fasting-induced suppression of the HPG axis in the adult male rhesus monkey (Macaca mulatta). Horm Metab Res. 2011;43(2):81-85. doi:10.1055/s-0030-1269852.

24. Tatone C, Carbone MC, Campanella G, et al. Female reproductive dysfunction during ageing: role of methylglyoxal in the formation of advanced glycation endproducts in ovaries of reproductively-aged mice. J Biol Regul Homeost Agents. 2010;24(1):63-72.

25. Codner E, Eyzaguirre FC, Iñiguez G, et al. Chilean group for the study of ovarian function in type, D. Ovulation rate in adolescents with type 1 diabetes mellitus.Fertil Steril. 2011;95(1):197-202. doi:10.1016/j.fertnstert.2010.10.041.

26. Roa J, Garcia-Galiano D, Varela L, Sanchez-Garrido MA, Pineda R, Castellano JM. The mammalian target of rapamycin as novel central regulator of puberty onset via modulation of hypothalamic Kiss1 system. Endocrinology. 2009;150:5016-5026.

27. Castellano JM, Navaro VM, Roa J, et al. Alterations in hypothalamic KiSS-1 system in experimental diabetes: early changes and functional consequences. Endocrinology. 2009;150:784-794.

28. Garcia-Galiano D, Pinilla L, Tena-Sempere M. Sex steroids and the control of the Kiss1 system: developmental roles and major regulatory actions. J Neuroendocrinol. 2012;24: 22-33.

29. Luque RM, Kineman RD, Tena-Sempere. Regulation of hypothalamic expression of KiSS-1 and GPR54 genes by metabolic factors: analyses using mouse models and a cell line. Endocrinology. 2007;148:4601-4611.

30. Livshits A, Seidman, DS. Fertility issues in women with diabetes. Womens Health. 2009;5:701-707.

31. Vahratian A, Barber JS, Lawrence JM, Kim C. Familyplanning practices among women with diabetes and overweight and obese women in the 2002 national survey for family growth. Diabetes Care. 2009;32(6)1026-1031.

32. Downs JS, Arslanian S, Bruin W, et al. Implications of type 2 diabetes on adolescent reproductive health risk. Diabetes Educ. 2010;36(6):911-919.

33. Iniguez G, Torrealba IM, Avila A, Cassorla F, Codner, E. Adiponectin serum levels and their relationships to androgen concentrations and ovarian volume during puberty in girls with type 1 diabetes mellitus. Hormone Res. 2008;70:112-117.

34. Codner E, Merino PM, Tena-Sempere M. Female reproduction and type 1 diabetes: from mechanisms to clinical findings. Hum Reprod. 2010;18(5):568-585.

35. Codner E, Cassorla F. Puberty and ovarian function in girls with type 1 diabetes mellitus. Hormone Research. 2007;71:12-21

36. Kim GL, Dhillon SS, Belsham DD. Kisspeptin directly regulates neuropeptide $\mathrm{Y}$ synthesis and secretion via the ERK1/2 and $\mathrm{p} 38$ mitogen-activated protein kinase signaling pathways in NPY-secreting hypothalamic neurons. Endocrinology. 2011;151:5038-5047.
37. Pal L, Chu HP, Shu J, Topalli I, Santoro N, Karkanias G. In vitro evidence of glucose-induced toxicity in $\mathrm{GnRH}$ secreting neurons: high glucose concentrations influence GnRH secretion, impair cell viability, and induce apoptosis in the GT1-1 neuronal cell line. Fertil Steril. 2007; 88:11431149.

38. Arrais RE, Dib SA. The hypothalamus-pituitary-ovary axis and type 1 diabetes mellitus: a mini review. Hum Reprod. 2006;21:327-337. DOI: 10.1093/humrep/dei353.

39. Moran A, Jacobs DR Jr, Steinberger J, et al. Changes in insulin resistance and cardiovascular risk during adolescence: establishment of differential risk in males and females. Circulation. 2008;117:2361-2368.

40. Codner E, Escobar-Morreale HF. Clinical review: hyperandrogenism and polycystic ovary syndrome in women with type 1 diabetes mellitus. J Clin Endocrinol Metab. 2007; 92:1209-1216.

41. Sirotkin AV. Growth factors controlling ovarian functions. J Cell Physiol. 2011;226:2222-2225.

42. Soto N1, Iñiguez G, López P, et al. Anti-Mullerian hormone and inhibin $\mathrm{B}$ levels as markers of premature ovarian aging and transition to menopause in type 1 diabetes mellitus. Hum Reprod. 2009;24(11):2838-44. doi: 10.1093/humrep/ dep276.

43. Diamanti-Kandarakis E, Piperi C, Korkolopoulou P, et al. Accumulation of dietary glycotoxins in the reproductive system of normal female rats. J Mol Med (Berl). 2007;85(12):1413-1420.

44. Lombardo F, Salzano G, Crisafulli G, et al. Menarcheal timing in intensively treated girls with type 1 diabetes mellitus. Nutrition. Nutr Metab Cardiovasc Dis. 2009;19(1):35-38. doi:10.1016/j.numecd.2007.12.002.

45. Picardi A, Cipponeri E, Bizzarri C, et al. Menarche in type 1 diabetes is still delayed despite good metabolic control. Fertil Steril. 2008;90(5):1875-1877. doi:10.1016/j. fertnstert.2007.09.035.

46. Pralong FP. Insulin and NPY pathways and the control of GnRH function and puberty onset. Mol Cell Endocrinol. 2010 Aug 5;324(1-2):82-86. doi:10.1016/j.mce.2010.01.037.

47. Deltsidou A, Lemonidou C, Zarikas V, Matziou V, Bartsocas CS. Oligomenorrhoea in adolescents with type 1 diabetes mellitus: relationship to glycaemic control. European Journal of Obstetrics, Gynecology \& Reproductive Biology. 2010;153:62-66.

48. Salonia A, Lanzi R, Scavini M, et al. Sexual function and endocrine profile in fertile women with type 1 diabetes. Diabetes Care. 2006;29:312-316

49. Rohrer T, Stierkorb E, Grabert M, et al. Delayed menarche in young German women with type 1 diabetes mellitus: recent results from the DPV diabetes documentation and quality management system. Eur J Pediatr. 2008;167:793799.

50. Zain M, Norman R. Impact of obesity on female fertility and fertility treatment. Future Medicine. 2008;4(2).183194.

51. Catalano PM. Obesity, insulin resistance, and pregnancy outcome. Reproduction. 2010;140;365-371.

52. Lu J, Zhao YY, Qiao J, Zhang HJ, Ge L, Wei Y. A followup study of women with a history of severe preeclampsia: relationship between metabolic syndrome and preeclampsia. Chin Med J (Engl). 2011;124(5):775-779.

53. Barton JR, Sibai BM. Prediction and prevention of recurrent preeclampsia. Obstet Gynecol. 2008;112:359-372. 
54. Erel CT, Senturk LM. The impact of body mass index on assisted reproduction. Current Opinion Obstetric Gynecology. 2009;21:228-235.

55. Maheshwari A, Stofberg L, Bhattacharya S. Effect of overweight and obesity on assisted reproductive technology: a systematic review. Human Reproduction Updates. 2007;13:433-444.

56. Metwally M, Ledger WL, LiTC. Reproductive endocrinology and clinical aspects of obesity in women. Ann N Y Acad Sci. 2008;1127:140-6. doi:10.1196/annals.1434.000.

57. Elbers CC, Charlotte N, Eijkemans MJ, Wijmenga C, Grobbee DE, Schouw, YT. Low fertility and the risk of type 2 diabetes in women. Human Reprod. 2010;26(12):34723478.

58. Wener-Ozegowska E, Zawiejska A, Michalowska-Wender G, Iciek R, Wender M, Brazert J. Metabolic Syndrome in Type 1 Diabetes Mellitus. Does It Have Any Impact On the Course of Pregnancy? J Physiol Pharmacol. 2011 Oct;62(5):567-73.

59. Samara-Boustani D, Colmenares A, Elie C, et al. High prevalence of hirsutism and menstrual disorders in obese adolescent girls and adolescent girls with type 1 diabetes mellitus despite different hormonal profiles. Eur J Endocrinol. 2012;166: 307-316.

60. Esposito K, Chiodini P, Colao A, Lenzi A, Giugliano D. Metabolic Syndrome and Risk of Cancer. Diabetes Care. 2012;35(11):2402-2411.

61. Harvey AE, Lashinger LM, Hursting S. The growing challenge of obesity and cancer: an inflammatory issue, Ann N Y Acad Sci. 2011;12(29):45-52.

62. Cust AE, Kaaks R, Friedenreich C, et al. Metabolic syndrome, plasma lipid, lipoprotein and glucose levels, and endometrial cancer risk in the European Prospective Investigation into Cancer and Nutrition (EPIC). Endocr Relat Cancer. 2007;14(3):755-767.

63. Kurdoglu Z, Ozkol H, Tuluce Y, Koyuncu I. Oxidative status and its relation with insulin resistance in young non-obese women with polycystic ovary syndrome. J Endocrinol Invest. 2012;35(3):317-321.

64. Zhang H, Pelzer AM, Kiang DT, Yee D. Down-regulation of type I insulin-like growth factor receptor increases sensitivity of breast cancer cells to insulin. Cancer Res. 2007;67(1):391-397.

65. Penranda EK, Shokar N, Ortiz M. Relationship between Metabolic Syndrome and History of Cervical Cancer among a US National Population. ISRN Oncol. 2013;2013:840964.

66. Barone BB, Yeh HC, Snyder CF, et al. Long-term all-cause mortality in cancer patients with preexisting diabetes mellitus: a systematic review and meta-analysis. JAMA.
2008;300:2754-2764.

67. Zhang Y, Liu Z, Yu X, et al. The association between metabolic abnormality and endometrial cancer: a large case-control study in China. Gynecol Oncol. 2010;117: 41-46. doi: 10.1016/j.ygyno.2009.12.029.

68. Rosato V, Zucchetto A, Bosetti C, Montella M, et al. Metabolic syndrome and endometrial cancer risk. Ann Oncol. 2011;22(4):884-889.

69. Lindemann K, Vatten LJ, Ellstrom-Engh M, Eskild A. Serum lipids and endometrial cancer risk: results from the HUNT-II study. Int J Cancer. 2009;124:2938-2941.

70. Friedenreich CM, Biel RK, Lau DC, et al. Case-control study of the metabolic syndrome and metabolic risk factors for endometrial cancer. Cancer Epidemiol Biomarkers Prev. 2011;20(11):2384-95. doi: 10.1158/1055-9965.EPI-11-0715.

71. Borena W, Stocks T, Jonsson H, et al. Serum triglycerides and cancer risk in the metabolic syndrome and cancer (Me-Can) collaborative study. Cancer Causes and Control. 2011;22(2);291-299.

72. Ulmer H, Borena W, Rapp K, et al. Serum triglyceride concentrations and cancer risk in a large cohort study in Austria. Br J Cancer. 2009;101(7):1202-1206.

73. Ronn R, Holzer HG. Oncofertility in Canada: the impact of cancer on fertility. Curr Oncol. 2013;20(4):338-344.

74. Whitworth KW, Baird DD, Stene LC, Skjaerven R, Longnecker MP. Fecundability among women with type 1 and type 2 diabetes in the Norwegian mother and child cohort study. Diabetologia. 2011;54:516-522.

75. Codner E, Iniguez G, Lopez P, et al. Metformin for the treatment of hyperandrogenism in adolescents with type 1 diabetes mellitus: a double-blind, randomized study. Horm Res. 2007;72:431.

76. Schweiger B, Klingensmith GJ, Snell-Bergeon JK. Menarchal timing in type 1 diabetes through the last 4 decades. Diabetes Care. 2010; 33: 2521-2523.

77. Lucenteforte E, Bosetti C, Talamini R, et al. Diabetes and endometrial cancer: effect modification by body weight, physical activity and hypertension. Br J Cancer. 2007;97: 995-998.

78. Samiksha S, Mahaveer PK, Natasha S, Dilip A, Gupta MK. Female infertility: an overview. IJPSR. 2011;2 (1):1-12.

79. Sjoberg L, Pitkaniemi J, Harjutsalo V, et al. Menopause in women with type 1 diabetes. Menopause. 2011;18:158-163.

80. Tena-Sempere, M. Roles of ghrelin and leptin in the control of reproductive function. Neuroendocrinology. 2007; 86:229-241.

81. Lindemann K, Vatten LJ, Ellstrom-Engh M, Eskild A. Serum lipids and endometrial cancer risk: results from the HUNT-II study. Int J Cancer. 2009;124:2938-2941.

Copyright (C) 2016 The Author(s); This is an open-access article distributed under the terms of the Creative Commons Attribution License (http://creativecommons.org/licenses/by/4.0), which permits unrestricted use, distribution, and reproduction in any medium, provided the original work is properly cited. 